\title{
Impact of Coronavirus Disease 2019 outbreak on acute coronary syndrome admissions: four weeks to reverse the trend
}

\author{
Marie Hauguel-Moreau ${ }^{1,2}$ - Rémy Pillière ${ }^{1}$ - Giulio Prati ${ }^{1}$ - Sébastien Beaune ${ }^{3}$ - Thomas Loeb ${ }^{4}$. Simon Lannou ${ }^{1,2}$. \\ Sophie Mallet ${ }^{1,2} \cdot$ Hazrije Mustafic $^{1,2} \cdot$ Céline Bégué $^{1}$. Olivier Dubourg ${ }^{1,2} \cdot$ Nicolas Mansencal $^{1,2}$
}

Published online: 29 June 2020

(c) Springer Science+Business Media, LLC, part of Springer Nature 2020

\begin{abstract}
Data whether the COVID-19 outbreak impacts the acute coronary syndromes (ACS) admissions and the time required to reverse the downward curve are scarce. We included all consecutive patients referred for an ACS who underwent PCI from February 17, 2020 to April 26, 2020 in a high-volume PCI coronary care unit. We compared the number of ACS patients in 2020 to the same period in 2018 and 2019. Predictors of adverse outcome in ST-elevation myocardial infarction (STEMI) patients were recorded: symptom-onset-to-first medical contact (FMC), and FMC-to-sheath insertion times. During the studied period (calendar weeks 8-17, 2018-2020), 144 ACS patients were included. In 2020, we observed two distinct phases in the ACS admissions: a first significant fall, with a relative reduction of $73 \%$, from the week of lockdown (week 12) to 3 weeks later and then an increase of ACS. Median symptom-onset-to-FMC time was significantly higher in 2020 than in the two previous years (600 $\mathrm{min}$ [298-632] versus $121 \mathrm{~min}$ [55-291], $\mathrm{p}<0.001)$. Median FMC-to-sheath insertion did not differ significantly (93 $\mathrm{min}$ [81-131] in 2020 versus $90 \mathrm{~min}$ [67-137] in 2018-2019, p=0.57). The main findings are (1) a pattern of a U-curve in ACS admissions, with a first decrease in ACS admissions and a return to "normality" 4 weeks after; (2) a significant increase in the total ischemic time exclusively due to an increase in the symptom-onset-to-first-medicalcontact time.
\end{abstract}

Keywords Acute coronary syndrome $\cdot$ ST-elevation myocardial infarction $\cdot$ COVID-19

\section{Highlights}

- During the COVID-19 pandemic, a dramatic decrease in ACS admissions was observed, followed by a return to "normality" one month later.

- In STEMI management, the ischemic time was more than threefold increased, exclusively due to an increase

Marie Hauguel-Moreau

marie.hauguel@aphp.fr

1 Department of Cardiology, Ambroise Paré Hospital (AP-HP), ACTION Study Group, Université de VersaillesSaint Quentin, Boulogne, France

2 INSERM U-1018, CESP, Épidémiologie clinique, Villejuif, France

3 Department of Emergency Medicine, Ambroise Paré Hospital, AP-HP, Boulogne-Billancourt, France

4 Service D’Aide Médicale D’Urgence (SAMU), Raymond Poincaré Hospital, APHP, Garches, France in patient-related time (symptom-onset-to-first medical contact time).

Since the outbreak of COVID-19 and the worldwide confinement, the number of acute coronary syndromes (ACS) dramatically decreased by $40 \%$ all around the world [1,2]. Data whether the COVID-19 outbreak impacts the ACS admissions and the time required to reverse the downward curve are scarce. We report a single center experience of patients hospitalized for ACS during the lockdown in a highvolume ( $>150$ primary percutaneous coronary intervention $[\mathrm{PCI}]$ per year) coronary care unit.

We included all consecutive patients referred for an ACS who underwent PCI from February 17, 2020 to April 26, 2020: 4 weeks before the lockdown (on March 17, 2020), 6 weeks after. We compared the number of ACS patients in 2020 to the same period in 2018 and 2019. Predictors of adverse outcome in ST-elevation myocardial infarction (STEMI) patients were recorded: symptom-onset-to-first medical contact (FMC), and FMC-to sheath insertion times. 


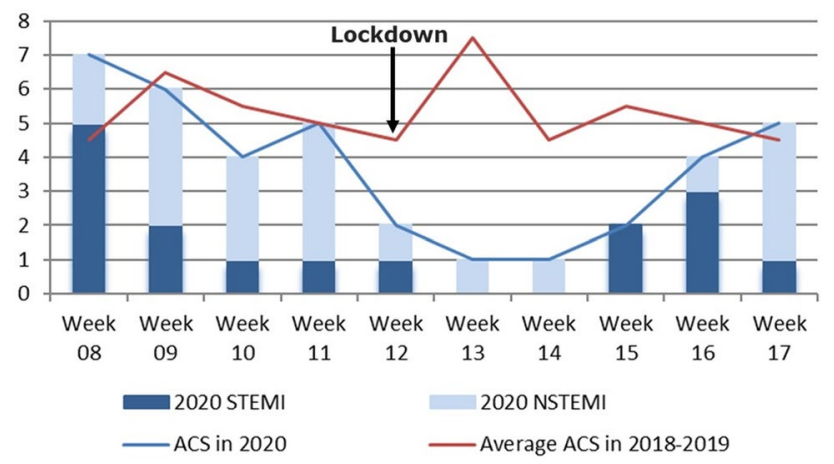

Fig. 1 Admissions of acute coronary syndrome during the Covid-19 pandemic period and in 2018-2019 from February 17 to April 26 (weeks 8-17). The COVID-19 lockdown was decided on March 17, 2020 in France (week 12)

During the studied period (calendar weeks 8-17, 2018-2020), 144 ACS patients were included. In 2020, 37 ACS patients underwent a PCI: 16 (43\%) were admitted for a STEMI versus 63 out of 107 (59\%) in 2018-2019 ( $p=0.03)$.

In 2020, we observed two distinct phases in the ACS admissions: a first significant fall, from the week of lockdown (week 12) to 3 weeks later (week 15) and then an increase of ACS (Fig. 1). As compared to 2018-2019 (weeks 12-15), a relative reduction of $73 \%$ in ACS admissions was observed. The same relative reduction of $73 \%$ was found comparing the 4 weeks before and after the lockdown.

During the lockdown (6 weeks), 6 STEMI patients were admitted, reflecting a relative reduction of $65 \%$, as compared to 2018-2019. In 2020, no STEMI was managed during the weeks 12-14, 2 and 3 STEMI within the weeks 15 and 16,1 within the week 17 . Median symptom-onset-to-FMC time was significantly higher in 2020 than in the two previous years (600 min [298-632] versus $121 \mathrm{~min}$ [55-291], $\mathrm{p}<0.001$ ). Median FMC-to-sheath insertion did not differ significantly (93 min [81-131] in 2020 versus $90 \mathrm{~min}$ [67-137] in 2018-2019, $\mathrm{p}=0.57$ ). In STEMI patients, mean left ventricular ejection fraction (LVEF) was $38 \pm 14 \%$ in 2020 versus $53 \pm 11 \%$ in $2018-2019(\mathrm{p}=0.009)$. In 2020 , one patient out of six developed an apical rupture and a cardiogenic shock (total ischemic time $=720 \mathrm{~min}$ ). One patient suffering from COVID-19 presented with ACS.

We found a pattern of U-curve, with a dramatic decline in ACS admissions with the beginning of the lockdown and, 1 month later, a relative return to normality. Lower pollution rates, lower daily stressors, more sleeping time, normal sympathetic activity recovery, less physical activity, less smoking (by fear of a worse pulmonary infection in case of COVID-19) have been initially proposed to explain this decreased number of ACS. Unfortunately, patients might also minimize symptoms and be reluctant to go to the hospital, by fear of COVID-19 infection.
The second main finding is the delay of STEMI management (three-fold increase in the ischemic time), exclusively due to a larger symptom-onset-to-FMC time, consistent with a previous study [3]. In our study, FMC-to-sheath insertion time was stable between years highlighting COVID-19 protective measures have no impact on STEMI management. Timely reperfusion by primary PCI is the standard treatment of STEMI patients. Delaying this time leads to a substantial increase of infarct-related morbidity and mortality. Two reasons were given by STEMI patients: fear of infection at the hospital (5 out of 6 patients) and avoidance of medical care by altruism (1/6). Once there is a similar pandemic in the future, physicians and media should communicate actively towards cardiac patients and general population on the need for urgent care if any unusual symptoms occur.

We report a single center experience and our findings should be validated in a larger cohort. However, during the COVID-19 pandemic, we observed a worrying early phase of reduction of ACS admissions with a significant increase in the total ischemic time in STEMI and, after 1 month, a second phase of recovery. If the reasons are not yet clear, effort should be done to make coronary patients warned again. The next challenge of physicians will be to manage simultaneously patients suffering from Covid-19 and patients suffering from acute or chronic disease.

Funding None.

\section{Compliance with ethical standards}

Conflict of interest No conflict of interests related to this manuscript.

\section{References}

1. Metzler B, Siostrzonek P, Binder RK, Bauer A, Reinstadler SJ (2020) Decline of acute coronary syndrome admissions in Austria since the outbreak of COVID-19: the pandemic response causes cardiac collateral damage. Eur Heart J 41:1852-1853

2. Garcia S, Albaghdadi MS, Meraj PM, Schmidt C, Garberich R, Jaffer FA, Dixon S, Rade JJ, Tannenbaum M, Chambers J et al (2020) Reduction in ST-segment elevation cardiac catheterization laboratory activations in the United States during COVID-19 pandemic. J Am Coll Cardiol 75:2871-2872

3. Tam CF, Cheung KS, Lam S, Wong A, Yung A, Sze M, Lam YM, Chan C, Tsang TC, Tsui M et al (2020) Impact of Coronavirus Disease 2019 (COVID-19) outbreak on ST-segment-elevation myocardial infarction care in Hong Kong, China. Circ Cardiovasc Qual Outcomes 13(4):e006631. https://doi.org/10.1161/CIRCO UTCOMES.120.006631

Publisher's Note Springer Nature remains neutral with regard to jurisdictional claims in published maps and institutional affiliations. 